\title{
Enhancement of diffusers BSDF Accuracy: Spectral Features Effect
}

\author{
Hedser van Brug ${ }^{1,2}$, Grégory Bazalgette Courrèges-Lacoste ${ }^{2}$, Gerard Otter ${ }^{2}$, Jos Groote \\ Schaarsberg ${ }^{2}$, Steven Delwart ${ }^{3}$ and Umberto del Bello ${ }^{3}$ \\ ${ }^{2}$ TNO Science and Industry, Stieltjesweg 1, 2628 CK Delft, the Netherlands \\ ${ }^{3}$ ESA ESTEC, Noordwijk, the Netherlands
}

\begin{abstract}
This paper reports the activities performed in the framework of the ESA contract 18432/04/NL/AR: Enhancement of diffusers BSDF Accuracy.

This study was conducted to investigate properties of various diffusers. Diffusers are widely used in space instruments as part of the on-board absolute calibration. Knowledge of the behavior of the diffuser is therefore most important.

From measurements of launched instruments in-orbit it has been discovered that when a diffuser is used in the vacuum of space the BSDF (Bi-directional Scattering Distribution Function) can change with respect to the one in ambient conditions. This is called the air/vacuum effect and has been simulated in this study by measuring the BSDF in a laboratory in ambient as well as vacuum conditions, results of this part of the study will be reported.

Another effect on the BSDF is not related to the air/vacuum effect, but to the design parameters of the optical system and the scattering properties of the diffuser. The effect is called Spectral Features and is a noise like structure superimposed on the BSDF. To observe this effect, spectral and spatial (partially) coherence light is needed. Highresolution spectrometers provide the spectral coherence and a narrow field of view provides the spatial coherence. Modern space spectrometers have high spectral resolution and/or a small field of view (high spatial resolution).

Different diffusers create different speckle patterns leading to different Spectral Features amplitudes. Therefore the choice of diffuser can be very critical with respect to the required absolute radiometric calibration of an instrument. Even if the Spectral Features are small it can influence the error budget of the retrieval algorithms for the level 2 products.

In this presentation diffuser trade-off results are presented and the Spectral Features model applied to the optical configuration of the MERIS instrument is compared to in-flight measurements of MERIS.

The introduction describes the use of diffusers in earth-observation satellites and why they cause spectral features. In Sec.2 the physical background of the spectral features, speckles, is discussed. Section 3 shows the results from air/vacuum effect measurements (SubSec.3.1), spectral features amplitude measurements on our in-house setup and simulations in a single graphical display (SubSec.3.2 ), and the measured and simulated results for the MERIS instrument (SubSec.3.3). The following sections deal with a description of the measuring setup and the model that has been made to do the simulations. Finally, in the discussion, topics like what is the best diffuser and what can be done to minimize the amplitude of the spectral features will be dealt with.
\end{abstract}

\section{Introduction}

Earth observing satellites for spectroscopic analysis of the earth atmosphere take the sun as illumination unit. The spectra recorded after passage through the atmosphere are calibrated using direct sun observations. To mimic the scattering of the earth, an on-board diffuser is included in the satellites. An additional function of this diffuser is to get a homogeneous illumination of the entrance slit of the spectrometer and to monitor the aging of the instrument.

In the past years a number of reports ${ }^{1,2}$ have been made about wiggles that are observed in the calibrated spectra of earth observation satellites that can not be due to absorption in the atmosphere. In the 2004 SPIE annual meeting a complete session was dedicated to the discussion of these wiggles. During that meeting we presented our research ${ }^{3}$ in this field. We have created a setup that is designed for testing diffusers and to get insight in the means to minimize the wiggle amplitudes. This setup will be presented in detail in this paper. The model to describe the wiggles was presented in 2004

\footnotetext{
${ }^{1}$ For further information please contact the author via: eddy.vanbrug@tno.nl
} 
and results obtained using this model were also discussed. Now we will repeat the basics of the model and give an extensive description of the results of the model and the experimental verification obtained using our setup. All presently known methods that can be used to reduce the amplitude of the wiggles will be presented.

In order to have a simple quantity to relate to a given diffuser, that tells directly the amplitudes of the wiggles that are to be expected, we propose the use of the Spectral Features Amplitude (SFA). Spectral Features are those features in the spectra that are due to the diffuser (and are not due to absorption effects in the earths atmosphere or due to emission lines in the spectrum of the light source). The standard deviation of the normalized data is taken as the SFA. The SFA can be calculated over a spectral width containing a few periods of spectral features, from the ratio of two independent measurements. Alternatively, the SFA can be calculated from a large number of independent spectra where the standard deviation is calculated per wavelength. Differently stated the Spectral Features Amplitude can be defined as: the deviation of the unwanted wiggles in the signal as measured in a spectrometer after eliminating all wanted features from the spectrum.

\section{Cause of Spectral Features}

When laser light (coherent or at least partially coherent $\left.{ }^{4}\right)$ is scattered by a rough surface, speckles are observed. This is due to interference effects that occur in all locations of an observation screen. Each location on that screen receives light from many points on the scattering object. All contributions can interfere as long as the path length differences are smaller than the coherence length of the light source.

In case of an earth observation satellite the light source in use is the sun. The coherence length of the light emitted by the sun is very short such that speckle effects are not easily observed. However, since the spectrometer limits the wavelength band per pixel, the light reaching each pixel will show interference effects and therefore also speckles. The effective coherence length $L_{c}$ for a spectral width of $\Delta \lambda$ and a central wavelength of $\lambda_{0}$ is obtained via

$$
L_{c}=\frac{\lambda_{0}^{2}}{\Delta \lambda} .
$$

Typical pixel bandwidth is about $0.5 \mathrm{~nm}$ in the visible, which gives an $\mathrm{L}_{\mathrm{c}}$ of about $500 \mu \mathrm{m}$. Typical path length differences for an aluminum diffuser are only a few microns, and thus far smaller than the coherence length per pixel.

Being a sensitive interference effect, the number of speckles present on each pixel changes with distances in the setup, illumination angle of the diffuser, etc. This means that the observed intensity per pixel will change over time. As a function of wavelength the intensity will even change for a perfectly stable setup. These intensity fluctuations with wavelength, that are due to changing speckle numbers and/or intensities, are what we refer to as Spectral Features. These Spectral Features can not be cancelled via a calibration scheme since they change with about everything. In a laboratory setup calibrating out the Spectral Features is sometimes feasible but in an operational satellite this is not an option.

The speckle size $\sigma_{\mathrm{s}}$ scales linearly with wavelength,

$$
\sigma_{s}=0.61 \frac{\lambda}{\mathrm{NA}},
$$

which means that the number of speckles falling onto a one dimensional pixel scales inversely proportional to the wavelength. The effect of the increase or decrease of the number of speckles in the detection area by one is stronger for a few speckles that when many speckles are present. This means that the Spectral Features will be more pronounced for longer wavelengths. The NA in Eq.(2) stands for numerical aperture of the optical system. In the case that a lens is used in the system the NA is defined by the aperture of the lens and the distance between lens and observation plane (in this paper this is taken to be the entrance slit of the spectrometer). If no lens is used, free space propagation, the NA is defined by the width of the scattering surface and the distance between diffuser and observation plane. 


\section{Results}

\subsection{Air/Vacuum effect}

The air/vacuum of the Spectralon diffuser was determined by measuring the BSDF for different angular configuration in both air and vacuum. For this a special vacuum chamber was developed to enable radiance and irradiance measurements within vacuum. Due to the nature of the chamber radiance could only be measured at fixed angles. The sum of the angle of incidence and the observed scattering angle is always 90 degrees.

A set of incidence and detection angles where measured under both ambient and vacuum conditions. The ratio between the ambient and the vacuum BSDF was used to determine any effect that might be present. In the figure below the results can be found. Note that the configuration 65 incidence/ 25 detection is measured twice. This configuration is measured as first and last of the series. Since the vacuum was fully established at the end the measurement are marked as vacuum 1 (beginning, poor vacuum) and 2 (end, good vacuum).

From Fig.1 it can be seen that there is no clear air vacuum behavior, although the ratios are significantly different from 1. The change of BSDF seems to be rather random. When the results are plotted against the pressure a more consistent picture can be seen. In figure 3 the results for $280 \mathrm{~nm}$ are given showing an air vacuum effect depending on the vacuum pressure. Only the point at 55/35 is off from the general trend. This is an unknown measurement artifact. For $410 \mathrm{~nm}$ and $1000 \mathrm{~nm}$ similar results are found only with smaller ratios (and no artifact at 55/35).

From the results the following was concluded:

- An air vacuum effect was observed for the Spectralon diffuser.

- The effect increases with decreasing wavelength, at $280 \mathrm{~nm}$ an effect of $1 \%$ was observed.

Relatif vacuum/air effect on the BRDF for all angles

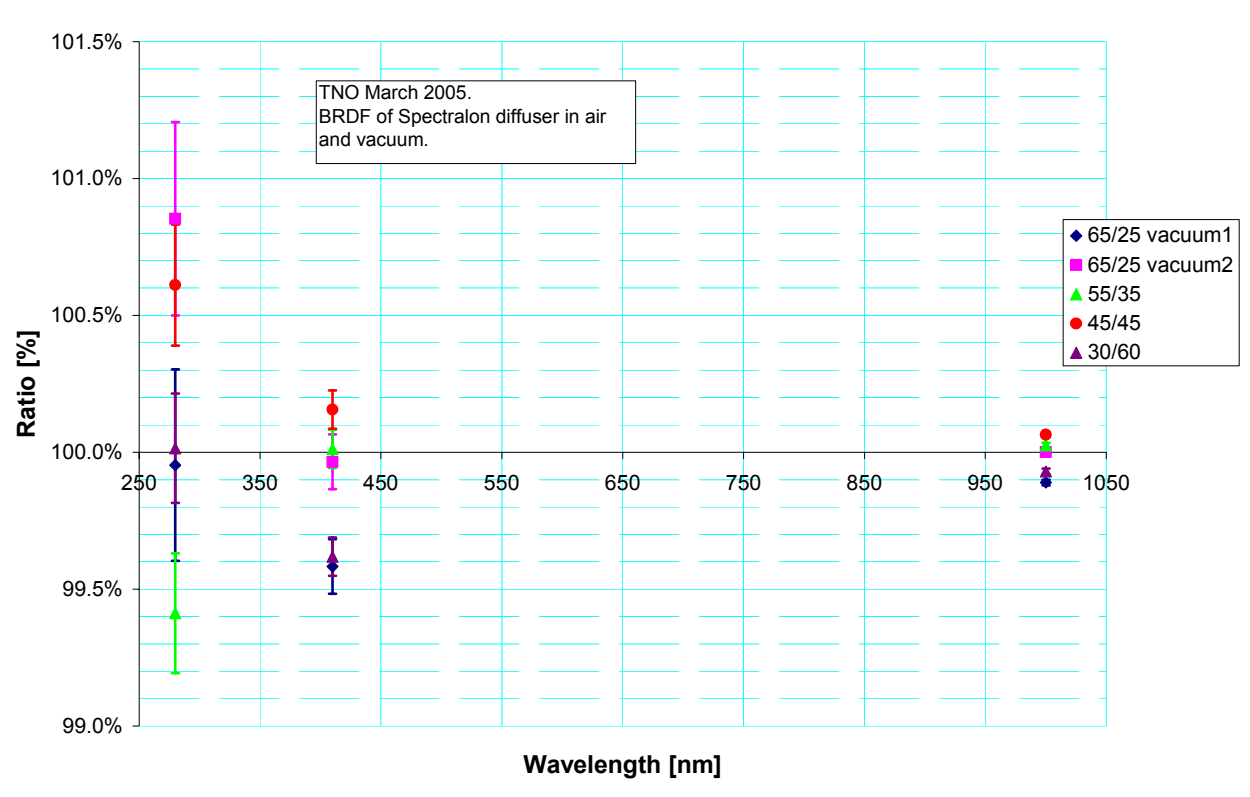

Figure 1 Ratio of the BSDF measurements in vacuum and air for all angles vs wavelengths. 
Relative air/vacuum effect on the BRDF versus pressure at $280 \mathrm{~nm}$

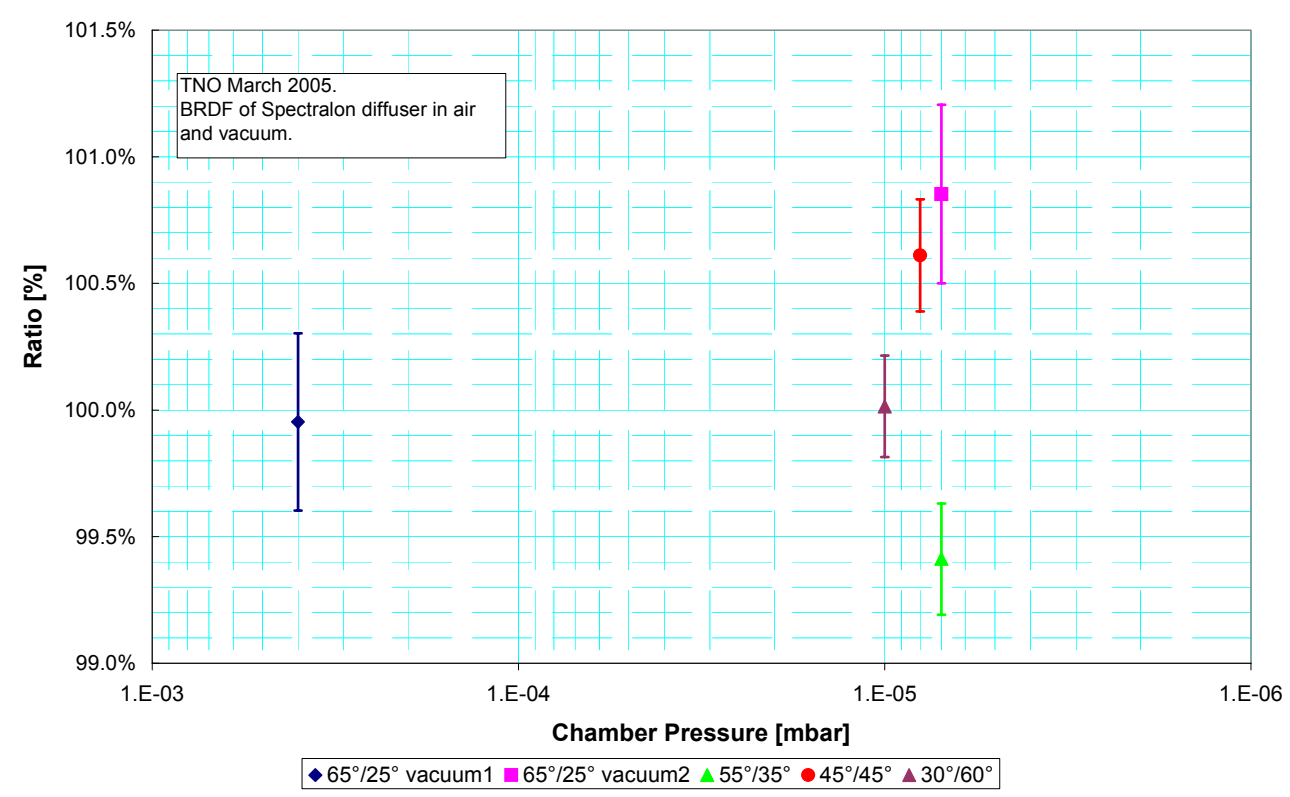

Figure 2 Ratio of the BRDF measurements in air and vacuum at $280 \mathrm{~nm}$ vs chamber pressure.

\subsection{Measured vs. simulated results: in-house setup}

In other publications normally the setup and model are presented before the results are shown. Since we want the outcome of the experiments to be known before the setup and modeling strategy are explained we have chosen to start with the presentation of the results.

This section shows some results of the measurement setup and compares them with the simulated results. For the simulation the configuration of the experimental setup was taken into account and the characteristics of each diffuser were used to get a realistic model. For the surface diffuser aluminum only the surface roughness had to be taken into account while for the volume diffuser Spectralon the penetration depth of the diffuser was also of importance. Finally, for the quasi volume diffuser (QVD) the separation between the two scattering surfaces and their roughness had to be taken into account for the model.

For all three diffuser SFA spectra, one feature can be seen (Figs.3 - 5) that is the same for all: the SFA is very low for wavelengths between 240 and $400 \mathrm{~nm}$ and then starts to rise strongly. This can be explained by the fact that the spectrometer consists of a prism for the first band $(240-400 \mathrm{~nm})$ and a grating for all longer wavelengths. This results in a poorer wavelength resolution in that first band which helps in reducing the SFA. 


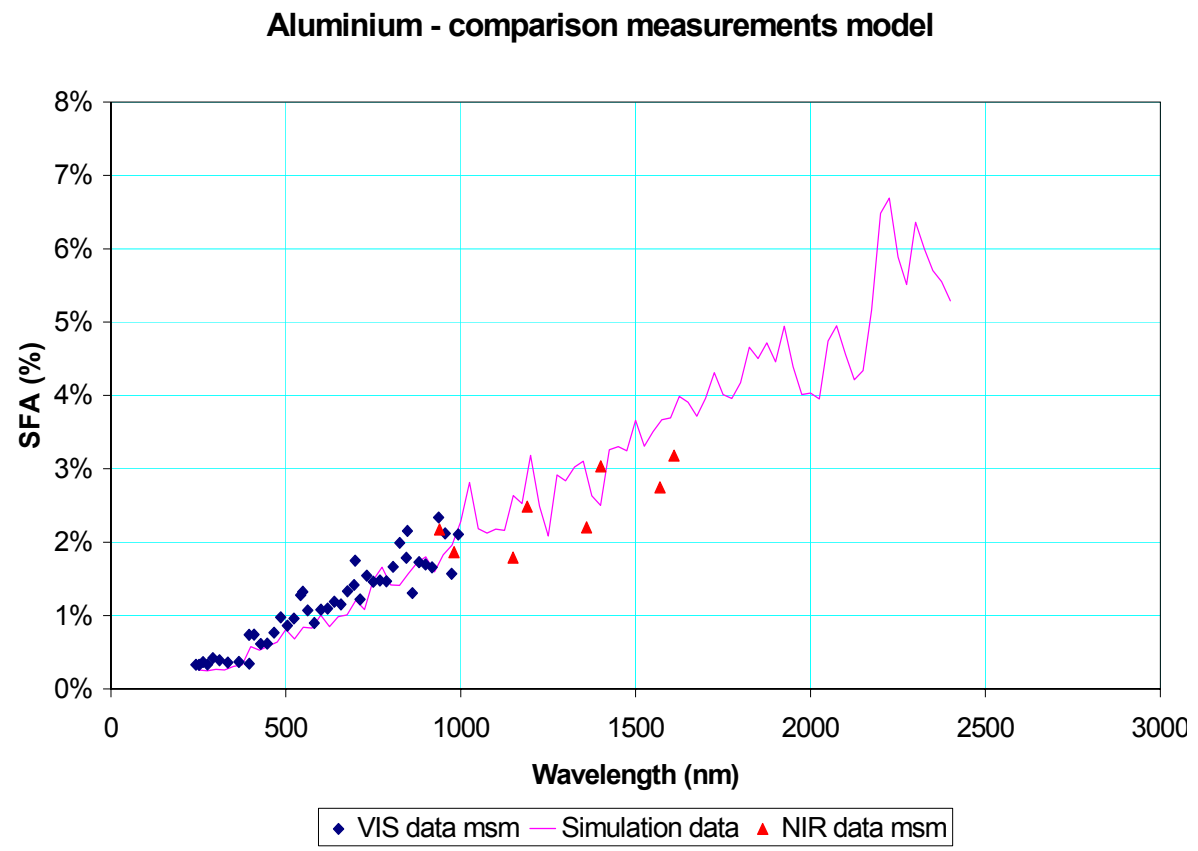

Figure 3 Comparison between the measured SFA for an Aluminum diffuser (points) and the data obtained using our simulation model (solid line)

QVD - comparison measurements model

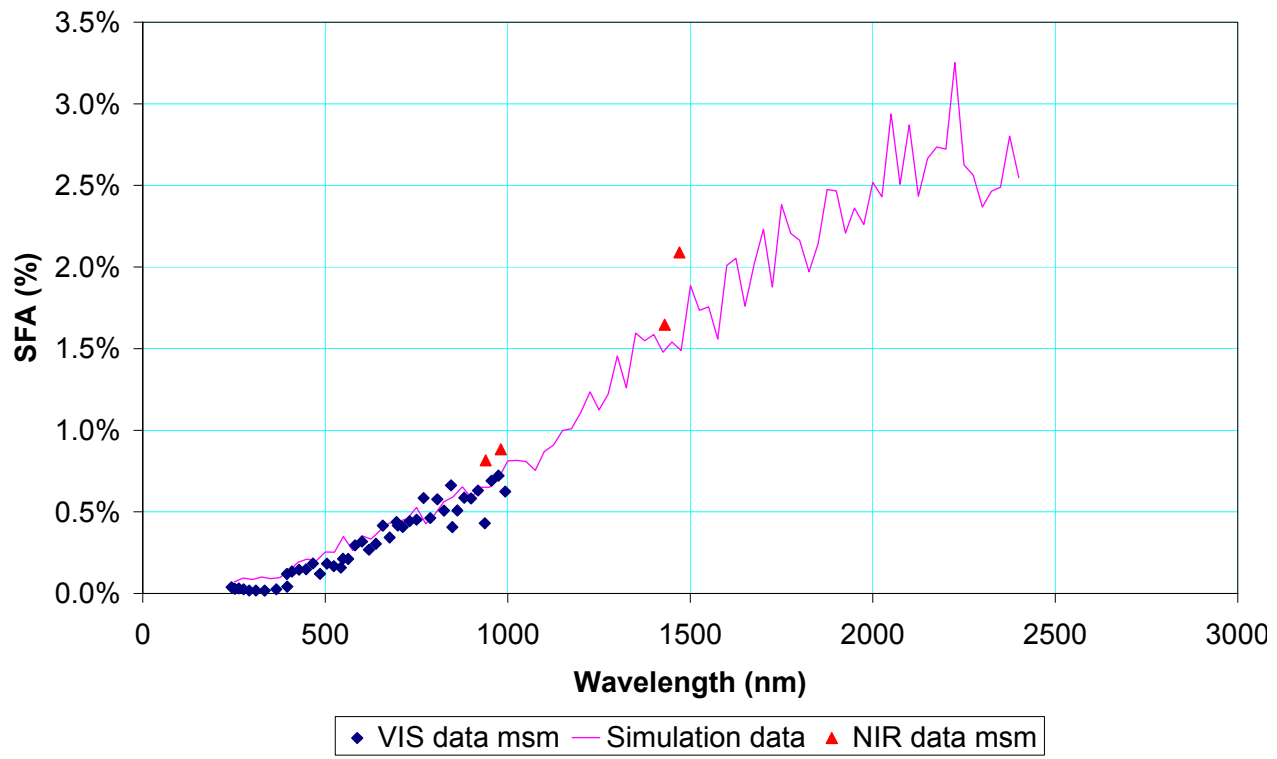

Figure 4 Comparison between the measured SFA for a quasi volume diffuser, QVD (points) and the data obtained using our simulation model (solid line) 


\section{Spectralon - comparison measurements model}

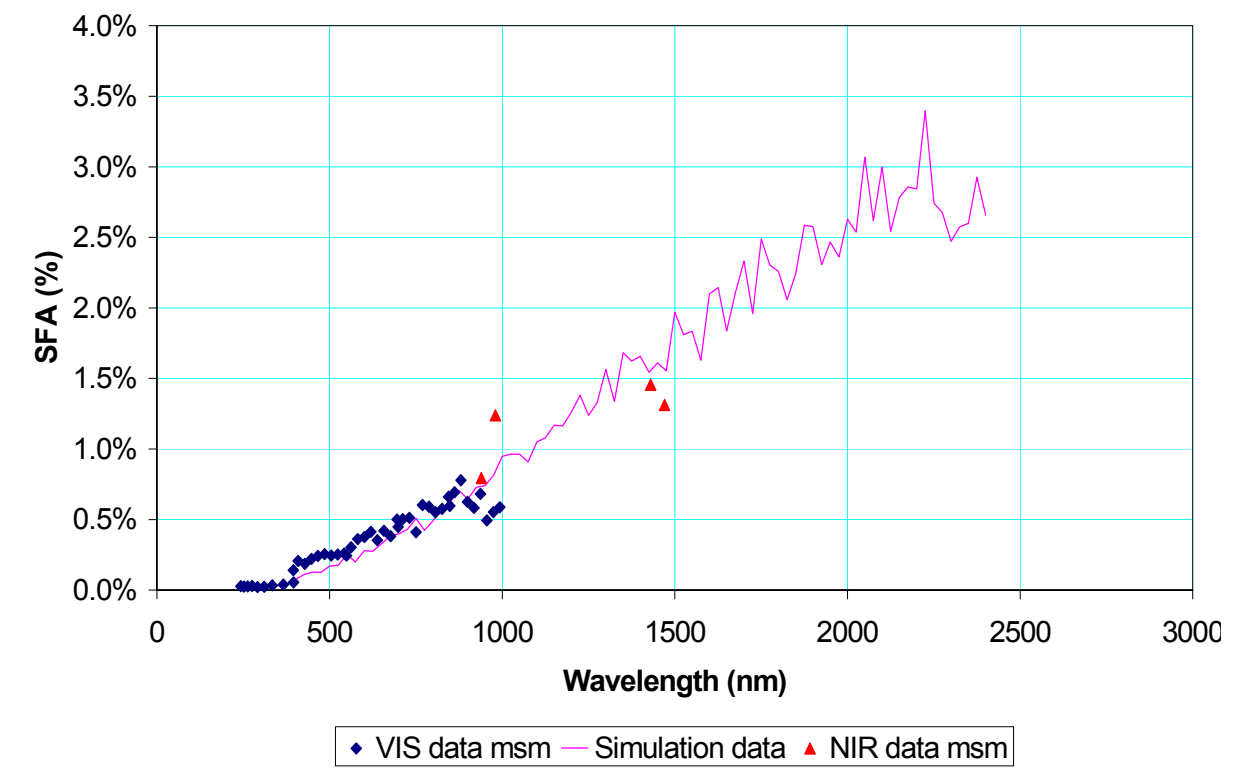

Figure 5 Comparison between the measured SFA for a Spectralon volume diffuser (points) and the data obtained using our simulation model (solid line)

\subsection{Measurement vs. simulated results: MERIS}

The experimentally obtained data from the MERIS instrument was given to us and we were asked to perform simulations to see what the capabilities are for this type of design for other wavelength regions. To show the level of agreement between simulations and actual data from a real live instrument the results are presented below.

In Fig.4 measured results from the MERIS instrument are presented (these data are NOT from our in-house setup, but from an actual instrument). The top graph in Fig.4 shows the data at $510 \mathrm{~nm}$, and the bottom graph for $900 \mathrm{~nm}$. The configuration of this instrument has been studied and implemented within a special version of our MatLab model. The outcome of the calculations are shown in Fig.5. The shown wavelength range is in accordance with the wavelengths used in MERIS 

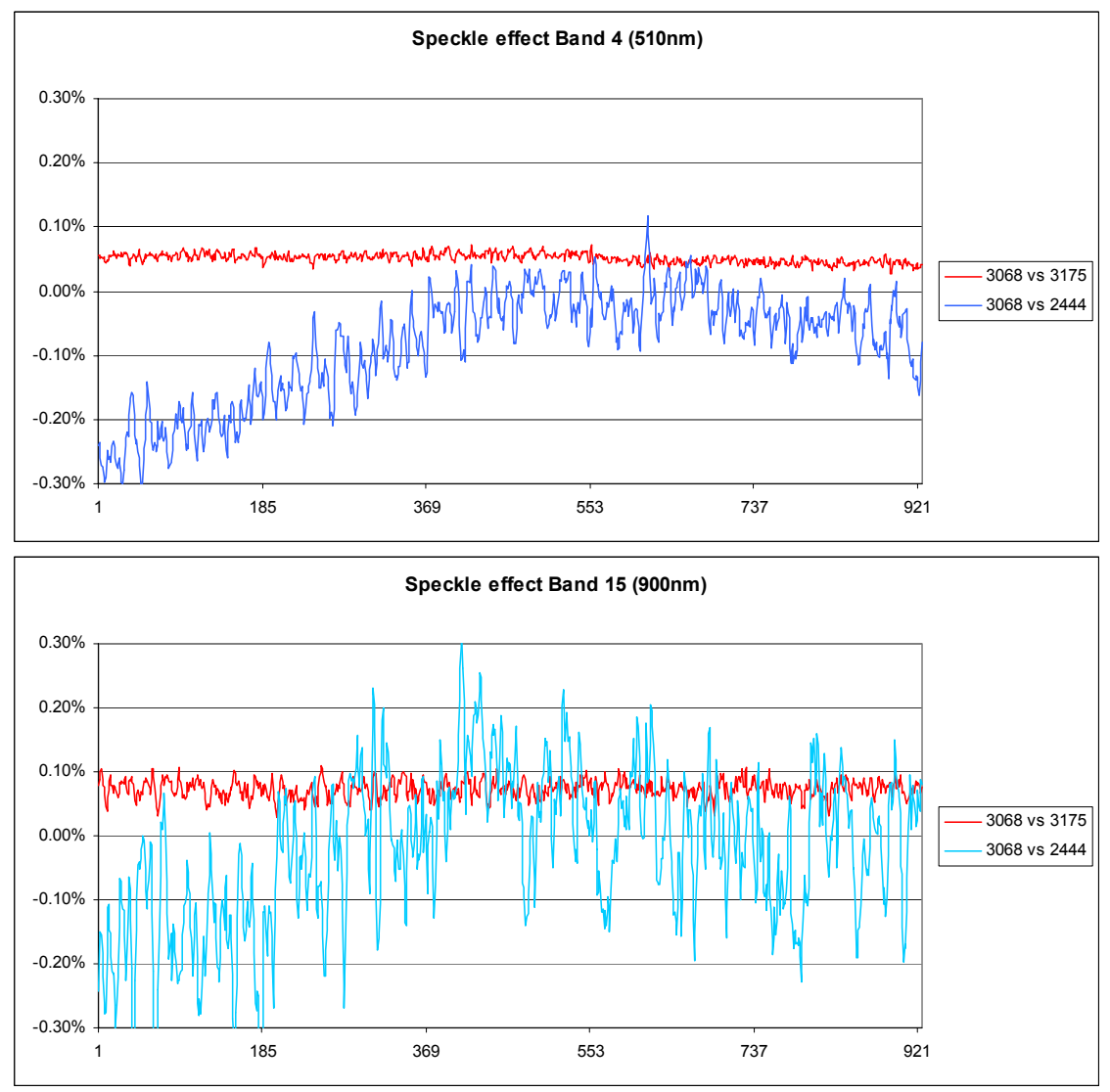

Figure 6 Observations made by the MERIS instrument for two different bands. The ratio is shown for a small sun angle difference (red) and a large sun angle difference (blue). 


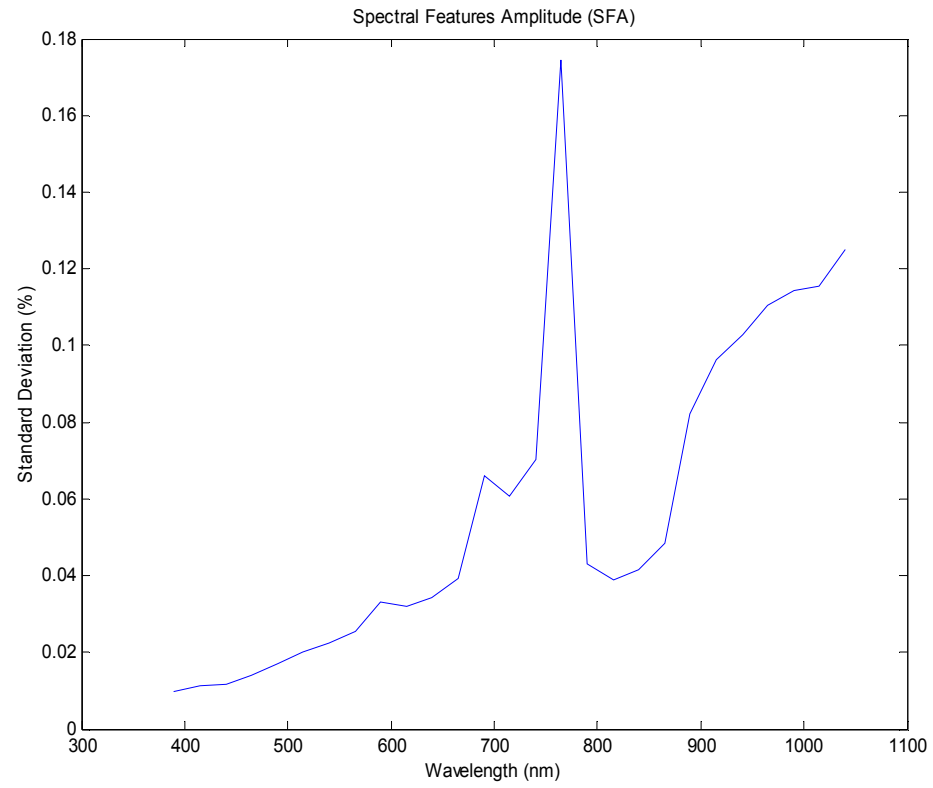

Figure 7 SFA calculations for the MERIS instrument

The measurements showed an SFA value of about $0.05 \%$ at $510 \mathrm{~nm}$ and about $0.1 \%$ at $900 \mathrm{~nm}$ (see Fig.6). The simulated SFA values for the whole wavelength range between $400 \mathrm{~nm}$ and $1050 \mathrm{~nm}$ are shown in Fig.5.

What is found is that the model is able to predict quantitatively the amplitude of the spectral features. The SFA values calculated with the model deviates from the MERIS observation by less than a factor 2 . This means that the model can be used to give a good prediction of what order of magnitude of SFA can be expected for an optical design.

Note that for the band around $760 \mathrm{~nm}$ the SFA curve has a strong peak. This peak corresponds with the smallest bandwidth of the corresponding band (i.e. $3.75 \mathrm{~nm}$ while the bandwidth of the other bands of MERIS is typically $10 \mathrm{~nm})$.

\section{Measuring setup and simulation model}

\subsection{Setup}

The components in the layout drawing (see Figure 8) of the dedicated Spectral Features measurement setup are numbered alphabetical:

A. Light source (QTH or Xenon type lamps)

B. Re-imaging aspheric mirror optics, to image the lamp onto $\mathrm{C}$

C. Pinhole/diaphragm (changeable size)

D. Collimation mirror (aspheric)

E. Diffuser or sample holder on top of stacked rotation and translation stages

F. Rotation table for light source and illumination optics

G. Spectrometer entrance optics (UV enhanced doublet to illuminate the entrance slit J)

H. Optional polarization module

I. Order filter wheel

J. Entrance slit of spectrometer

K. Collimation mirror

L. Turret containing the diffractive optics (prism and grating)

M. Detector unit ('Hamamatsu' CCD array)

N. Darkened area

O. Imaging mirror 
This paper only gives an overview of the components present in the setup. For further information on the setup please contact the authors. The illumination unit is build on a rotation stage allowing us to change the angle of incidence on the diffuser. The diffuser itself is also placed on a, separate, rotation stage allowing the change of angle under which the diffuser is seen by the spectrometer. Between the diffuser and the entrance slit of the spectrometer a lens is placed such that our system is in the so called mixed mode. A mixed mode is a mode where the 'imaging' from the diffuser onto the entrance slit consists of two or more of the following pure modes. The first pure mode is Free Space Mode. In this case no optical elements are used for the imaging. The second pure mode is the Imaging Mode. In the imaging mode the diffuser is imaged onto the entrance slit via a lens. The third and final pure mode is the Fourier Mode. Here the diffuser is placed in the front focal plane of the lens and the entrance slit in the back focal plane. In our setup we have a mix between Free Space Mode and Fourier Mode. The diffuser stands at about half a meter away from the front focal plane of the lens. The entrance slit is in the back focal plane.

The actual spectrometer consists of two types of dispersive elements. The first is a prism that is used for the lowest wavelength band (Band I: $240-400 \mathrm{~nm}$ ). The other wavelength bands are measured with a grating as dispersive element (Band II: $390-550 \mathrm{~nm}$, Band III: $540-700 \mathrm{~nm}$, Band IV: $690-850 \mathrm{~nm}$, and finally Band V: $840-999 \mathrm{~nm}$ ). All these bands are measured using a 1024 pixels Si CCD detector. After replacing this detector with a 512 pixels InGaAs CCD detector also some near infrared bands can be measured, using a differently blazed grating to optimize the throughput in the NIR. The NIR bands (Bands VI through XV) are half the spectral width of the bands in the visible in order to arrive at similar spectral widths per pixel. The wavelengths measured in the NIR range from 920 through $1630 \mathrm{~nm}$.

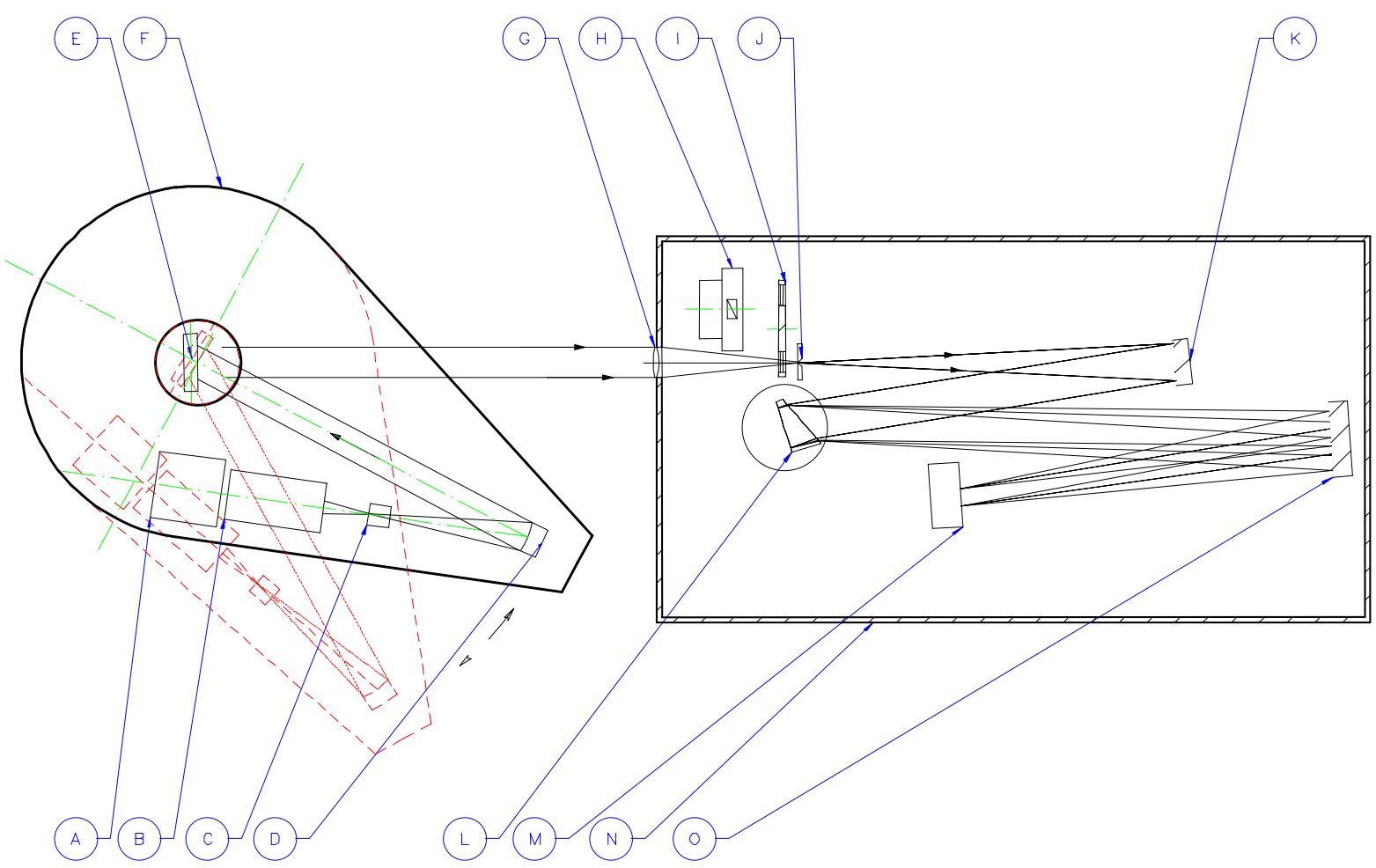

Figure 8 Layout of the dedicated Spectral Features setup

The optics inside the spectrometer were designed such that the numerical aperture of the beam illuminating the entrance slit is smaller than the acceptance angles of the mirrors labeled $\mathrm{K}$ and $\mathrm{O}$ in Fig.1. In this way the number of speckles in the entrance slit is the relevant number is the spectral features calculations. All optical elements inside the spectrometer (except for the prism of course) are used in reflection to exclude chromatic effects. 


\subsection{Model}

A MatLab program has been written to simulate the spectral features effects. Two version exist: a $1 \mathrm{D}$ version and a 2D version. The $2 \mathrm{D}$ version can be used to simulate the actual optical arrangement with as major drawback the time issue. A single simulation takes several days. To overcome this problem a $1 \mathrm{D}$ version was constructed with which a single simulations can be done within hours and for which it was found that the results are in good to excellent agreement with measured data.

A 1D program is useable owing to the fact that the entrance slit of the spectrometer is far longer than it is wide. In the smallest direction the number of speckles can be taken as one due to the size of the speckle with respect to the entrance slit, but more importantly due to the dispersion effect caused by the grating or prism in the spectrometer. In case it is expected that the number of speckles in the shortest direction of the entrance slit is larger than unity this can easily be compensated for in the software. This compensation can be made wavelength dependent using theoretical scaling laws.

Each run of the model always starts with defining a rough surface. At some distance away from this surface an 'image' is formed either by imaging using a lens, free propagation, or as Fourier transform also by using a lens. Several of these stages can be combined to describe the actual optical arrangement. After all these stages the image at the entrance slit of the spectrometer is obtained. Separate runs have to be made for all wavelengths. In the entrance slit the intensity is calculated such that the intensity as a function of the wavelength is obtained. In a real instrument the entrance slit is imaged onto a detector (array or matrix detector) via the spectrometer. By following the presented approach we effectively model a one to one imaging spectrometer.

The description so far explains how the intensity (for each wavelength) is calculated at the entrance slit of the spectrometer. The steps that follow this primary calculation are: a) taking into account the type and specifications of the diffuser, b) taking into account the spectral width of each detector element, and finally c) taking into account the opening angle of the illumination source (for earth observation satellites very often the sun). Each of these three steps will be elaborated upon the following.

a) Type of diffuser: for a surface diffuser like aluminum only a single speckle pattern is created at the entrance slit. Owing to the fact that QVD and Spectralon have a certain thickness that is far larger than the coherence length of the light a number of independent speckle images are formed at the entrance slit. The higher the number of independent images the lower the contrast in the resulting speckle image will be, leading to a reduction in spectral features amplitude. The coherence length of the light scales with the wavelength. This means that the spectral feature reduction is largest for short wavelengths.

b) Spectral width of a detector pixel: apart from the amplitude of the spectral features they also have a characteristic period. If a large number of periods is present within the entrance slit the intensity will be averaged and the resulting spectral features will be reduced. This effect is referred to as "inner pixel averaging". The spectral feature period increases with wavelength such that again the reduction is largest for shorter wavelengths.

c) Opening angle of illumination source: the speckle pattern created in the entrance slit changes strongly with changes in the illumination conditions. This means that many independent speckle images are created when a large opening angle is used. These independent images will again result in a reduction of the spectral features.

After all these steps the final spectral features effect can be calculated. To get a single figure to indicate the effect we have proposed the SFA: the Spectral Features Amplitude. The SFA is defined as the standard deviation from the ratio between two spectra (that are recorded under slightly different circumstances). The described numeric approach yields the spectra. Two or more spectra are calculated each with its own diffuser definition (hereby simulating the change in angle or shift of the diffuser). The ratio between two consecutively calculated spectra is determined and from that ratio the standard deviation is determined, i.e. the SFA.

\section{Discussion}

So far it has been indicated that our model agrees very good with real measurements, both for the in-house spectral features testing facility and for a real live instrument. This section will give a short resume of techniques that can be used to reduce the SFA value. 


\subsection{Angular extent}

The angular extent of the light source used yields a number of independent speckle patterns. The extent of the sun is for instance already 0.5 degrees. The speckle patterns are independent when their contributions do not interfere and when the speckle patterns have no correlation. This mechanism for SFA reduction is always present but can be optimized by choosing the proper diffuser and optical arrangement.

\subsection{Number of speckles per detector pixel}

The SFA scales inversely proportional with the number of speckles per pixel. This means that increasing the size of the pixels, or reducing the size of the speckles will help reducing the SFA. The width of the entrance slit can not be changed freely since it is one of the factors that determine the resolution of the spectrometer. The optical arrangement is therefore the only key to reduce the speckle size and therewith increase the number of speckles per detector pixel. The length of the slit can be used to influence the amount of speckles per pixel. This length can not always be chosen freely since it is sometimes used for spatially resolved data and then the length influences the spatial resolution that can be obtained.

\subsection{Polarization}

Since two orthogonally polarized beams can not interfere, these two beams will result in two independent speckle patterns. This will yield a reduction by the square root of two. Since the polarization state of the light source is in general a given fact the only extra reduction that can be achieved is by choosing a diffuser that will scramble the polarization state. It general it can be stated that volume diffusers have this scrambling capability.

\subsection{Movements}

The speckle pattern will change strongly by a translation and/or rotation of the diffuser. These movements are therefore a very useful tool to reduce the SFA. If during a recording of the spectra the diffuser is being moved, the speckle pattern will smear out and will thereby be reduced in contrast. This is a very strong method to reduce the SFA. The drawback is that an actuator has to be implemented in the setup. In orbit this can be realized by using a long measuring time while the satellite moves with respect to the sun.

\subsection{Pixel bandwidth}

The spectral features have apart from their amplitude also a period. It was found that depending on the mode in which the diffuser is used the period can be smaller than the spectral bandwidth of the detector pixels. This leads to an averaging within the pixels. This averaging method can be optimized by increasing the bandwidth of the pixels. This is directly a degradation of the achieved resolution and therefore not a good approach. The alternative is to optimize the optical arrangement to decrease the period of the spectral features.

\subsection{Diffuser}

The type of diffuser is the most straightforward means to influence the spectral features. An aluminum diffuser will in general produce far stronger features than a volume diffuser or a QVD. QVD is in particular very good in reducing spectral features for illumination sources with a 'large' opening angle. Both QVD and the real volume diffusers can be used advantageously for small coherence lengths. These coherence lengths pertain to the bandwidth for each pixel on the detector.

\subsection{Optical arrangement}

That the optical arrangement can be optimized to reduce the SFA is more or less obvious. This arrangement dictates the size of the speckles and hence the number of speckles per detector pixel. It also defines the period of the spectral features and therewith the averaging within a detector pixel. And finally the averaging due to the opening angle of the illumination source is, amongst others, determined by this arrangement.

\section{Conclusions}

The air-vacuum experiments show that the BRDF for Spectralon changes with pressure and that the change is larger for smaller wavelengths. 
It has been shown that spectral features can be fully explained by speckle theory and that speckles have therefore to be seen as the source of spectral features. A single quantity has been introduced, the SFA (spectral features amplitude), that can be used to arrange different diffusers in terms of spectral features reduction capabilities. According to the SFA values it can be stated that QVD is the best diffuser, Spectralon is very similar in performance to QVD, and that an aluminum diffuser performs clearly worse (by a factor of three for the presented in-house spectral features testing setup).

The spectral features setup has been presented together with results obtained using this setup. The MatLab program that has been written for the modeling of spectral features has been discussed and results using this program are shown to be in agreement with the measured results, for three different diffusers.

In the final section a summary has been given for all methods that can be used to reduce the spectral features.

As stated above QVD is found to perform best. Spectralon is about equal but that is owing to the fact that Spectralon has a polarization scrambling effect. This means that for polarized operation QVD is clearly the best diffuser.

As final conclusion we can state that from the presented research we have learned enough about speckles and their effects in spectrometers that it is now possible to predict the amount of spectral features once an optical design for a spectrometer is known, and that we can optimize the optical design for future spectrometers in order to reduce the spectral features amplitude to below an acceptable level.

\section{Acknowledgment}

This work was funded by the European Space Agency (ESA).

\section{REFERENCES}

1. A. Richter, F. Wittrock, A. Ladstätter-Weißenmayer, J. Burrows "GOME measurements of stratospheric and tropospheric BrO", Advanced Space Research 29 (11), 1667-1672 (2002)

2. B. Ahlers, G. Bazalgette Courrèges-Lacoste, C. Schrijvers, and H. van Brug, "In-orbit detection of Spectral Features in SCIAMACHY", In: Sensors, Systems, and Next-Generation Satellites VIII, Proc. SPIE 5570, Maspalomas, Spain, September 13-16, pp.401-410 (2004)

3. H. van Brug, R. Vink, J. Groote Schaarsberg, G. Bazalgette Courrèges-Lacoste, and B. Snijders, "Speckles and their effects in spectrometers due to on-board diffusers", In : Earth Observing System IX, Proc. SPIE 5542, Denver, Colorado USA, August 2-6, pp. 334-341(2004)

4. H. Fujii and T. Asakura, A contrast variation of image intensity under illumination of partially coherent light", Optics Communications 12 (1), 32-38 (1974) 